\title{
Perirhinal Cortex Ablation Impairs Visual Object Identification
}

\author{
Mark J. Buckley and David Gaffan \\ Department of Experimental Psychology, Oxford University, Oxford, OX1 3UD, United Kingdom
}

\begin{abstract}
Impairments in both recognition memory and concurrent discrimination learning have been shown to follow perirhinal cortex ablation in the monkey. The pattern of these impairments is consistent with the hypothesis that the perirhinal cortex has a role in the visual identification of objects. In this study we compared the performance of a group of three cynomolgus monkeys with bilateral perirhinal cortex ablation with that of a group of three normal controls in two tasks designed to test this hypothesis more directly. In experiment 1 the subjects relearned a set of 40 familiar concurrent discrimination problems; the stimuli in each trial were digitized images of real objects presented in one of three different views. After attaining criterion they were tested on the same problems using similar, but previously unseen, views of the objects. In experiment 2 the subjects were tested on their ability to perform 10 of these
\end{abstract}

familiar discriminations with each problem presented in the unfamiliar context of a digitized image of a unique complex scene. The subjects with ablations were significantly impaired on both tasks. These results demonstrate that the role of the perirhinal cortex is not restricted to memory, and they support the hypothesis that the perirhinal cortex is involved in visual object identification. We suggest that the perirhinal cortex is crucially involved in processing coherent concepts of individual objects. A deficit of this nature could underlie the pattern of impairments that follow perirhinal cortex damage in both visual object recognition memory and visual associative memory.

Key words: monkey; macaque; learning; memory; object identification; perirhinal cortex; TE; inferior temporal cortex; temporal lobe
It is well established that visual recognition memory in the monkey, as tested by delayed matching and nonmatching to sample (DMS and DNMS) with trial-unique objects, is severely impaired after combined damage to the perirhinal and entorhinal cortices or after damage to the perirhinal cortex alone (Murray et al., 1989; Gaffan and Murray, 1992; Meunier et al., 1993; Eacott et al., 1994). In contrast, after combined perirhinal and entorhinal cortex ablation, impairments were not revealed in new postoperative concurrent discrimination learning with $24 \mathrm{hr}$ intertrial intervals (Gaffan and Murray, 1992; Eacott et al., 1994). This appeared consistent with the hypothesis that concurrent discrimination learning engaged a corticostriatal habit system, whereas DNMS engaged a limbic memory system (Malamut et al., 1984; Mishkin and Petri, 1984; Overman et al., 1990). However, recent studies have demonstrated that concurrent discrimination learning is in fact impaired after perirhinal cortex ablation alone (Buckley and Gaffan, 1997, 1998a). Because both fornix transection (Gaffan, 1992) and amygdalectomy (Gaffan, 1994a) have also been shown to impair discrimination learning with $24 \mathrm{hr}$ intertrial intervals, it is now evident that the limbic system is involved in such learning. Thus, the perirhinal cortex can no longer be regarded as either a structure primarily involved in short- as opposed to long-term memory or as a structure primarily involved in memory as opposed to habit. Clearly, the role of the perirhinal cortex in the primate's visual learning and memory system needs to be reassessed in light of the new behavioral data.

The impairments in new concurrent discrimination learning that were shown to follow perirhinal cortex ablation were revealed using manipulations to the basic task, which either in-

Received Aug. 14, 1997; revised Dec. 22, 1997; accepted Jan. 6, 1998.

This research was supported by the Medical Research Council (UK).

Correspondence should be addressed to Mark J. Buckley, Department of Experimental Psychology, Oxford University, South Parks Road, Oxford, OX1 3UD, UK.

Copyright (C) 1998 Society for Neuroscience $0270-6474 / 98 / 182268-08 \$ 05.00 / 0$ creased the number of incorrect choices in each trial, increased the number of problems in each set, or displayed objects in different views (Buckley and Gaffan, 1997, 1998a). In addition, although DMS with large stimulus sets was severely impaired after combined perirhinal and entorhinal cortex ablation, DMS with small sets was not (Eacott et al., 1994). Therefore, we suggested previously that the perirhinal cortex plays a role in the visual identification of objects, because in both types of task impairments were revealed only when the burden placed on the subject's ability to identify multiple individual objects was sufficiently heavy.

To test this hypothesis, in experiment 1 a group of monkeys with perirhinal cortex ablations (PRh) and a group of normal controls $(\mathrm{CON})$ learned a 40-problem concurrent discrimination learning task to criterion using digitized images of familiar views of each object. They were subsequently tested on the same problems using similar but previously unseen views. If perirhinal cortex ablation impairs object identification, then we would predict that the PRh group would be impaired when identifying new views of familiar objects. In experiment 2 the two groups of monkeys were required to relearn 10 of these problems concurrently, this time with the objects presented in the context of a digitized image of a complex scene. Likewise, if perirhinal cortex ablation impairs object identification, we would predict that the $\mathrm{PRh}$ group would be impaired when identifying familiar objects presented in the unfamiliar context of a complex scene.

\section{MATERIALS AND METHODS}

Subjects

Six male cynomolgus monkeys (Macaca fascicularis) took part in this experiment. They were housed either individually or in pairs, in rooms with automatically regulated lighting, and they were given water ad libitum. Before the experiments reported here, three subjects had the perirhinal cortex ablated bilaterally (PRh), whereas the other three subjects served as unoperated controls (CON). All six monkeys had 
identical pre- and postoperative experience in color discrimination and concurrent discrimination learning tasks in a series of experiments that were performed before the present study commenced, and they also completed a configural learning task and a paired associate learning task between the two experiments reported here (Buckley et al., 1997; Buckley and Gaffan, 1997, 1998a,b).

\section{Surgery}

The operations were performed in sterile conditions with the aid of an operating microscope, and the monkeys were anesthetized throughout surgery with barbiturate (5\% thiopentone sodium solution) administered through an intravenous cannula. The zygomatic arch was removed, and the temporal muscle was detached from the cranium and retracted. Surgery was performed on one hemisphere at a time. A bone flap was raised over the frontal and temporal lobe. The medial and posterior limits of the flap were in a crescent shape extending from within $5 \mathrm{~mm}$ of the midline at the brow to the posterior insertion of the zygoma. The anterior limit of the flap was the brow and the orbit. Ventrally the flap extended from the posterior insertion of the zygoma to the level of the superior temporal sulcus in the lateral wall of the temporal fossa anteriorly. The ventral anterior part of this bone flap was extended with a rongeur to the base of the temporal fossa. The dura mater was cut to expose the dorsolateral frontal and lateral temporal lobe. The frontal lobe was retracted from the orbit with a brain spoon to enable access to the anterior medial temporal lobe. Pia mater was cauterized, and the underlying cortex was removed by aspiration in the lateral bank of the anterior part of the rhinal sulcus and in the adjacent $2 \mathrm{~mm}$ of cortex on the third temporal convolution. The monkey's head was then tilted to an angle of $120^{\circ}$ from the vertical, and the base of the temporal lobe was retracted from the floor of the temporal fossa with a brain spoon. The posterior tip of the first part of the ablation was identified visually, and the removal was then extended in the lateral bank of the rhinal sulcus to the posterior tip of the sulcus, again removing $2 \mathrm{~mm}$ of laterally adjacent tissue. The dura mater was then sewn, the bone flap was replaced, and the wound was closed in layers. This ablation was made in both hemispheres in a single operation.

\section{Histology}

After the conclusion of all behavioral experiments, the animals with ablations were sedated, deeply anesthetized, and then perfused through the heart with saline solution $(0.9 \%)$, which was followed by formol saline solution ( $10 \%$ formalin in $0.9 \%$ saline solution). The brains were blocked in the coronal stereotaxic plane posterior to the lunate sulcus, removed from the skull, allowed to sink in sucrose formalin solution (30\% sucrose, $10 \%$ formalin), and sectioned coronally at $50 \mu \mathrm{m}$ on a freezing microtome. Every tenth section through the temporal lobe was stained with cresyl violet and mounted. Figure 1 shows, for each monkey, five of these sections spaced $4 \mathrm{~mm}$ apart through the lesioned area. Figure 2 shows the extent of the intended lesion on a labeled ventral illustration of a standard monkey brain with an illustration of the actual extents of the lesions in two representative subjects. Further detailed drawings of reconstructions of these lesions have also been published elsewhere (Buckley et al., 1997). The extent of the perirhinal lesions in all three cases was essentially as intended, except for some inadvertent damage caused by slight involvement of the laterally adjacent area TE (on the left in PRh1 and PRh3 and bilaterally in PRh2), for an anteroposterior extent of 2 and $4 \mathrm{~mm}$ on the left and right sides, respectively. There was slight involvement of the most lateral part of the entorhinal cortex in PRh2 and $\mathrm{PRh} 3$ and slight involvement of the anterior parahippocampal cortex in all three cases.

\section{Apparatus and stimuli}

The present tasks were performed in an automated test apparatus. The subject sat in a wheeled transport cage fixed in position in front of a touch-sensitive screen $(380 \times 280 \mathrm{~mm})$ on which the stimuli could be displayed. The subject could reach out between the horizontal or vertical bars (150 mm apart) at the front of the transport cage to touch the screen. An automated pellet delivery system, controlled by the computer, delivered reward pellets into a food well that was $80 \mathrm{~mm}$ in diameter and was positioned in front of and to the right of the subject. Reward pellets (190 $\mathrm{mg}$ ) were only delivered in response to a correct choice made by the subject to the touch screen. Pellet delivery was accompanied by an audible click. An automated lunchbox (length $200 \mathrm{~mm}$, width $100 \mathrm{~mm}$, height $100 \mathrm{~mm}$ ) was positioned in front of and to the left of the subject. The lunchbox was spring-loaded and opened immediately with a loud crack on completion of the whole task. The lunchbox contained the subject's daily diet of cereals, seeds, proprietary primate pellets, nuts, raisins, and half an apple or banana. An infrared camera was positioned looking down into the transport cage from above to allow the subject to be observed while it was engaged in the task. The whole apparatus was housed in an experimental cubicle that was dark except for a $25 \mathrm{~W}$ incandescent lamp. This lamp was positioned on the floor, below the level of the touch screen, to avoid any reflection onto the screen but to allow the subject to see into the cup and lunchbox when the screen was dark. The presentation of the visual stimuli on the touchscreen was controlled by a computer. The computer also recorded the responses that subjects made to the touchscreen and controlled the delivery of reward pellets after correct responses, and it controlled the opening of the lunchbox after completion of the session.

The stimulus material for task 1 was composed of digitized images of real objects presented on a touchscreen. To create these digitized images, each object was photographed in front of a plain white or black background using an electronic camera (Canon ION, model RC-260). Six different views of each object were taken. For each of two elevations $\left(\sim 30\right.$ and $\left.60^{\circ}\right)$ looking down on the object, three different rotations were photographed (face on, $45^{\circ}$ rotated left, and $45^{\circ}$ rotated right). The images were then saved as 8 bit, 256 color, bitmap (BMP) files with a spatial resolution of $368 \times 272$ pixels. Each image was then reduced to 64 colors using a Stuki dither color reduction algorithm. A computer program analyzed each of these files and converted the background of each image into a homogenous plain gray color. The BMP files were finally converted into a format that could be used by a separate program that was designed to display two of these images on the screen instantaneously, side by side, on a plain gray background. The resolution of the display was $1024 \times 768$ pixels.

The stimulus material for task 2 was composed of digitized images of scenes containing varying numbers of foreground and background objects. To create these digitized images, each scene was photographed using the Canon ION electronic camera. A total of 10 different scenes were selected. In addition to the other foreground and background objects contained in the scene, each scene also contained a pair of foreground objects; one was positioned on the right and the other on the left of the scene. Each scene was photographed with this pair of objects placed in their respective foreground positions, with their positions reversed, and with both objects on the same side. This latter contingency allowed a section of the scene containing the object to be alternated with the same section of the photograph of the scene with the object absent, at the same time maintaining full color balance in the scene as a whole, thus allowing objects to be flashed on and off within the scene as visual feedback, as required during the task. The images were then saved as 8 bit, 256 color, BMP files with a spatial resolution of $736 \times 544$ pixels. Each image was then reduced to 128 colors using a Stuki dither color reduction algorithm. The BMP files were finally converted into a format that could be used by a separate program that was designed to display one of these scenes on the screen instantaneously. The same program also allowed sections of photographs of the same scene to be rapidly and instantaneously alternated, as described above, to give the impression of a flashing stimulus object as required. The resolution of the display was $800 \times 600$ pixels.

\section{Behavioral testing}

Experiment 1: identification of familiar objects presented in new views. Experiment 1 consisted of two stages. In stage A the subjects learned to criterion a concurrent discrimination learning task consisting of 40 pairs of digitized images of objects, with each object presented on the touchscreen in one of three different views in each trial. In stage B the subjects were retested in the same manner using one of three similar but previously unseen views of the same objects. No preliminary training was given in the automated apparatus because the subjects were experienced in touching stimuli on the touchscreen to obtain food reward.

Stage $A$. The subjects were required to learn concurrently a set of 40 pairs of object discriminations between pairs of digitized images of objects presented on a plain gray background on a touchscreen. These 40 pairs of objects were familiar to the subjects because they had already learned concurrent discrimination learning tasks to criterion with these stimuli in a previous study in which only three out of the six different views of each object were used (Buckley and Gaffan, 1998a). These three digitized images of different views were chosen at random with respect to the two levels of elevation but included one image with the object face on, one image with the object rotated $45^{\circ}$ to the left, and one image with 

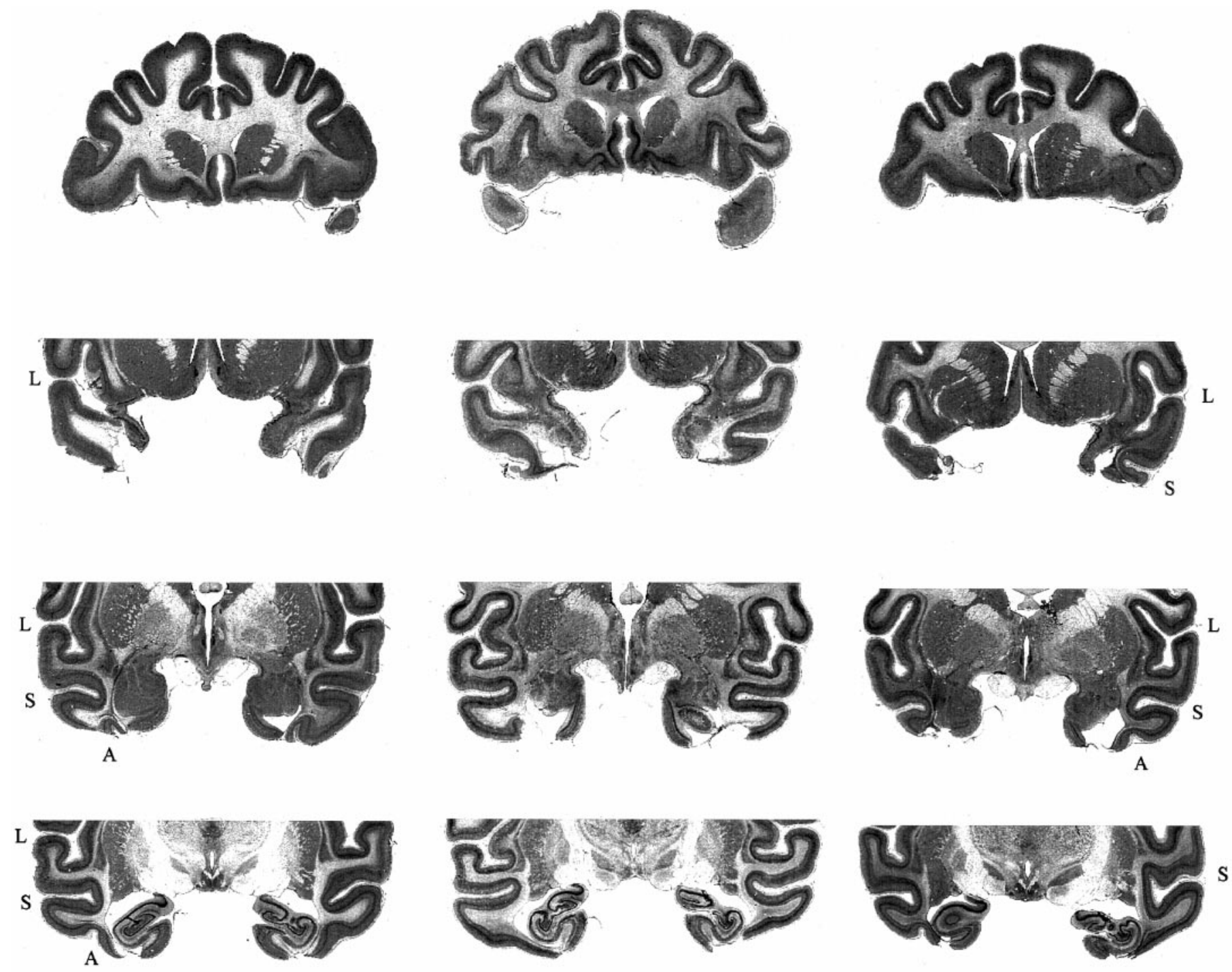

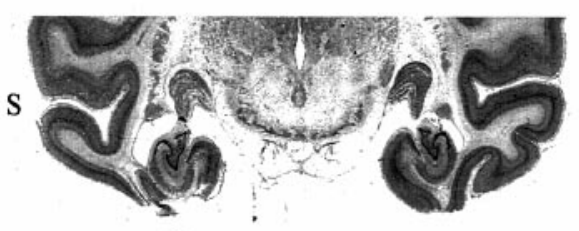

$\mathrm{O}$

PRh1

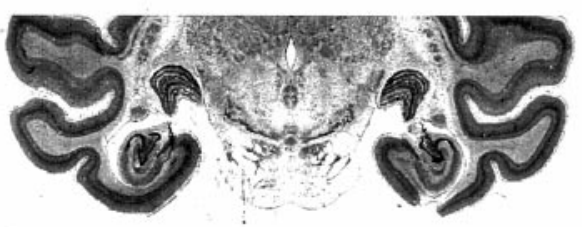

PRh2

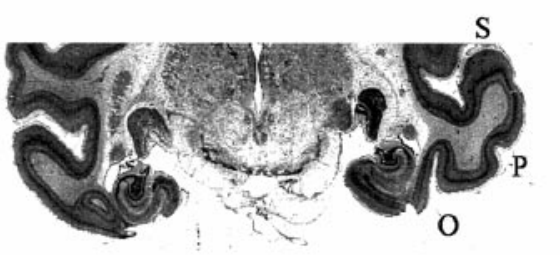

PRh3

Figure 1. Five $50 \mu \mathrm{m}$ sections of the brain are shown for each animal in the perirhinal groups (PRh1, $P R h 2, P R h 3)$. From top to bottom the sections are spaced $4 \mathrm{~mm}$ apart running anterior to posterior through the area of the bilateral perirhinal cortex ablation. $L$, Lateral sulcus; $S$, superior temporal sulcus; $A$, anterior middle temporal sulcus; $P$, posterior middle temporal sulcus; $O$, occipital sulcus.

the object rotated $45^{\circ}$ to the right. The object-reward associations that were used in the last stage of this previous study were maintained in stage A of the present study, and only the same three views of each object that were used in this previous study were used in stage $\mathrm{A}$ of the present study. The left-right position of the correct choice $(\mathrm{S}+)$ and incorrect choice $(\mathrm{S}-)$ on the touchscreen was randomized from trial to trial. Which of the three different views of each object that would be presented in each trial was determined pseudorandomly before the session began. Figure 3 illustrates three representative problems between pairs of digitized images as they would appear on the touchscreen (top row) and shows how the view of each object may change in a subsequent presen- tation of the same problem (bottom row). If the subject touched the $\mathrm{S}+$, then a reward pellet was delivered, the $\mathrm{S}-$ disappeared, and the $\mathrm{S}+$ remained on the screen for a further $1.5 \mathrm{sec}$ before the intertrial interval of $10 \mathrm{sec}$ commenced. If the subject touched the $\mathrm{S}-$, both the $\mathrm{S}+$ and $\mathrm{S}-$ disappeared and the intertrial interval of $10 \mathrm{sec}$ commenced. There were no correction trials. If the subject touched elsewhere on the screen, both images remained until one of them was touched. If the subject touched the screen during the $10 \mathrm{sec}$ intertrial interval, then the $10 \mathrm{sec}$ intertrial interval was restarted from that time. In each session the set of 40 problems was repeated three times and in the same sequence. After all 120 trials within the session had been completed, the automated lunchbox 


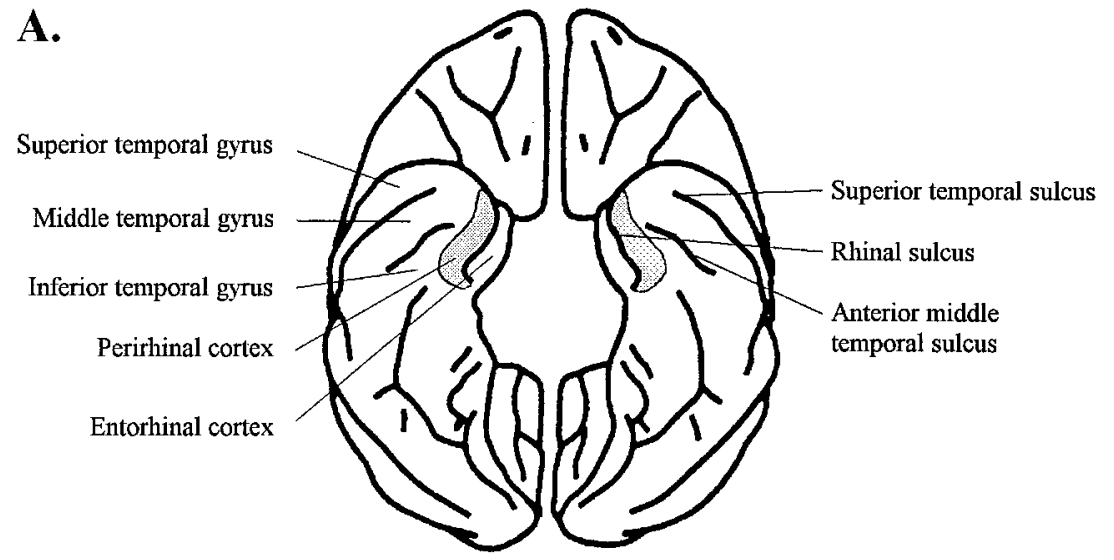

Intended extent of lesion Actual extent of lesion

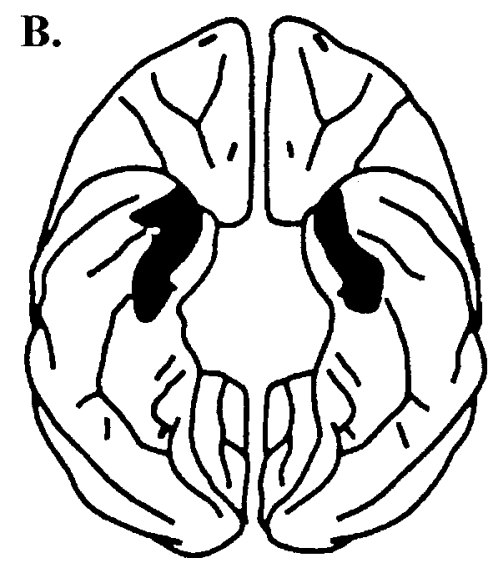

PRh1

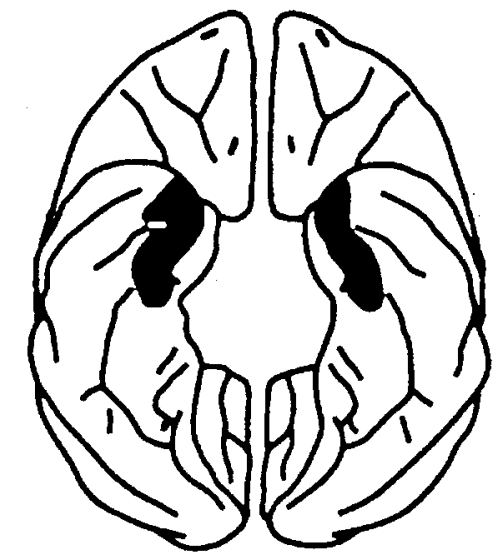

PRh3
Figure 2. A, Shaded regions show the intended location and extent of the ablation of the perirhinal cortex on a schematic diagram of the ventral view of the brain with sulci and gyri in labeled regions; $B$, shaded regions show the extent of the actual perirhinal cortex lesions in two representative subjects $(P R h 1, P R h 3)$. opened immediately to make available the subject's daily meal, as described above. The subjects performed one session of this task every day until they attained a criterion of $\geq 90 \%$ correct responses made within a single session. On the next day after attaining criterion, they proceeded to stage B of experiment 1

Stage B. In stage B the subjects continued testing on the same problems in the same order and in the same manner as in stage A. The only difference was that instead of using the views of the objects that had been used in stage A, in each trial of stage B the objects were presented in one of the three remaining views that until this stage of this experiment had never been seen before by the subjects. The subjects performed one session of this task every day until they attained a criterion of $\geq 90 \%$ correct responses made within a single session

Experiment 2: identification of familiar objects presented in scenes. In experiment 2 the subjects learned to criterion a concurrent discrimination learning task consisting of 10 problems. Each of these problems consisted of a unique scene that contained two objects that were designated as the $\mathrm{S}+$ and $\mathrm{S}-$ in addition to various other foreground and background objects. Each of the $10 \mathrm{~S}+$ and $\mathrm{S}-$ object pairs were a pair of objects that had been used in experiment 1 and also in a previous study (Buckley and Gaffan, 1998a). The object-reward contingencies remained unchanged for each of these 10 pairs of objects. The subjects therefore were highly familiar with these objects and with the object-reward contingencies; however, they had not experienced previously the objects in the context of a scene. Each pair of objects was always presented within the same scene but the left/right position of the objects in the foreground of the scene was chosen pseudorandomly before the trial began. The top row of Figure 4 illustrates three representative problems between pairs of objects presented in their scenes as they would appear on the touchscreen; the bottom row of
Figure 4 shows the same problem with the $S+$ and $S-$ positions reversed. If the subject touched the $\mathrm{S}+$, then a reward pellet was delivered and the $\mathrm{S}+$ flashed on and off within the scene five times, providing visual feedback for a correct response before the intertrial interval of $15 \mathrm{sec}$ commenced. If the subject touched the $\mathrm{S}-$, then the screen blanked and the intertrial interval of $15 \mathrm{sec}$ commenced. There were no correction trials. If the subject touched elsewhere on the screen than the $\mathrm{S}+$ or $\mathrm{S}-$, then the scene remained on the screen until either the $\mathrm{S}+$ or $\mathrm{S}-$ was touched. If the subject touched the screen during the $15 \mathrm{sec}$ intertrial interval, then the $15 \mathrm{sec}$ intertrial interval was restarted from that time. The subjects were first required to learn problems one to five concurrently. The subjects performed one session of this task every day until they attained criterion of $\geq 90 \%$ correct responses made within a single session. In each of these sessions the set of five problems was repeated in the same sequence until the subject had made 100 correct responses. On the next day after attaining criterion on problems one to five, the subjects were required to learn problems 6 to 10 concurrently. The subjects performed one session of this task every day until they attained criterion of $\geq 90 \%$ correct responses made within a single session. In each of these sessions the set of five problems was repeated in the same sequence until the subject had made 100 correct responses. On the next day after attaining criterion on problems 6 to 10 , the subjects were required to learn problems 1 to 10 concurrently. The subjects performed one session of this task every day until they attained criterion of $\geq 90 \%$ correct responses made within a single session. In each of these sessions, the set of 10 problems was repeated in the same sequence until the subject had made 100 correct responses. In all of these sessions, when the 100th correct response was made the automated lunchbox opened immediately to make available the subject's daily meal, as described above. 


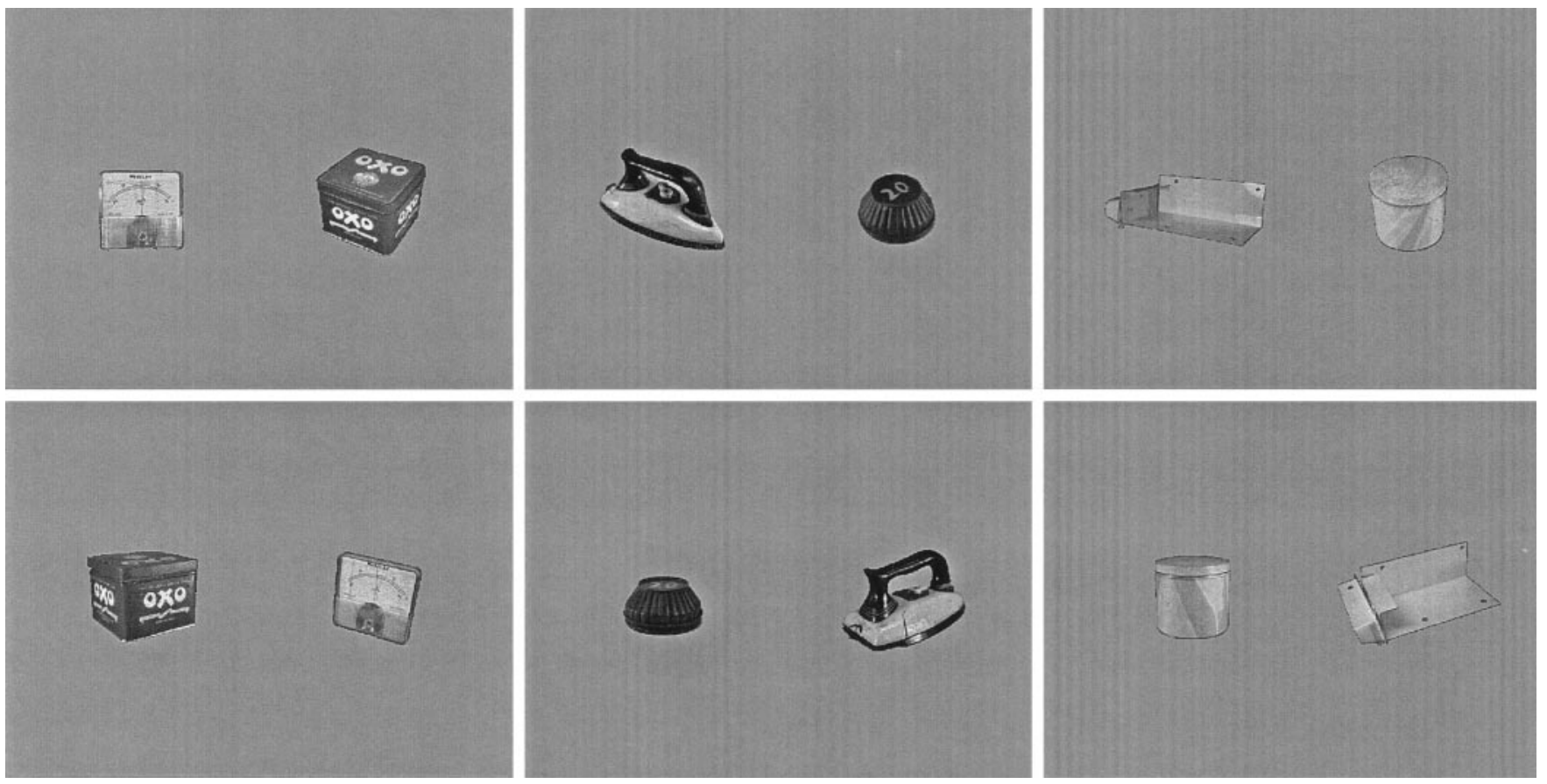

Figure 3. Examples of how three different discrimination problems from experiment 1 appear to subjects (reproduced in gray scale) when stimuli are presented as digitized images of objects on the touchscreen. For each of three different problems the figure illustrates how the appearance of the objects changes with presentation of the objects in different views.
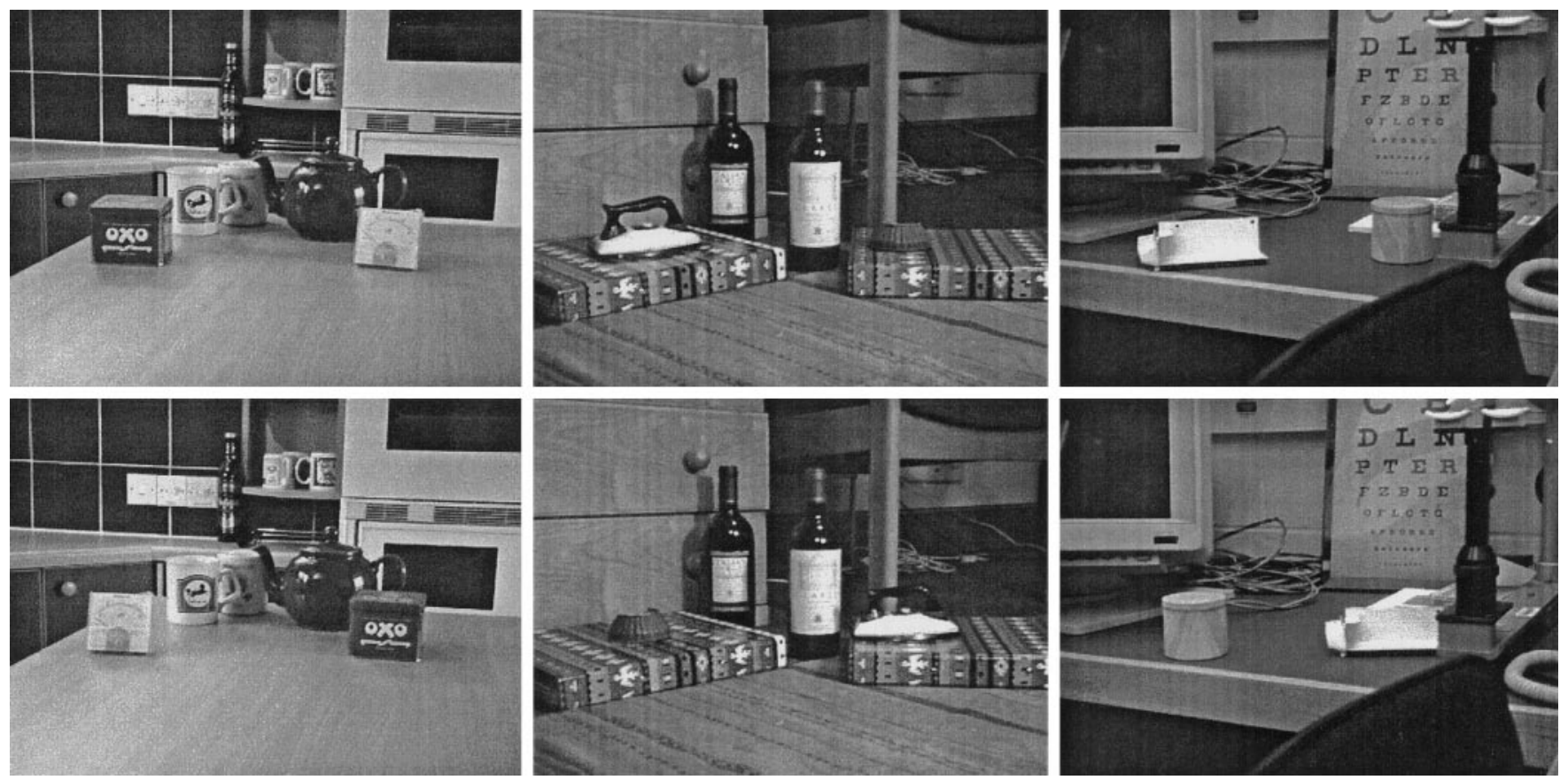

Figure 4. Examples of how three different discrimination problems from experiment 2 appear to subjects (reproduced in gray scale) when stimuli are presented in the context of digitized images of unique complex scenes. For each of three different problems the figure illustrates how the scene appears with the positions of the $\mathrm{S}+$ and $\mathrm{S}-$ reversed.

\section{RESULTS}

\section{Experiment 1: identification of familiar objects presented in new views}

In stage $\mathrm{A}$ the subjects were required to learn 40 concurrent discrimination problems to criterion with digitized images of objects presented in one of three different views. The $\mathrm{CON}$ group made a mean of 120 errors before attaining criterion in stage A, whereas the PRh group made a mean of 214 errors. After attaining criterion in stage A the subjects were tested on the same problems in stage B but with the objects presented in similar but previously unseen views. Errors to criterion from stage B in which the subjects had to identify familiar objects in new views are 


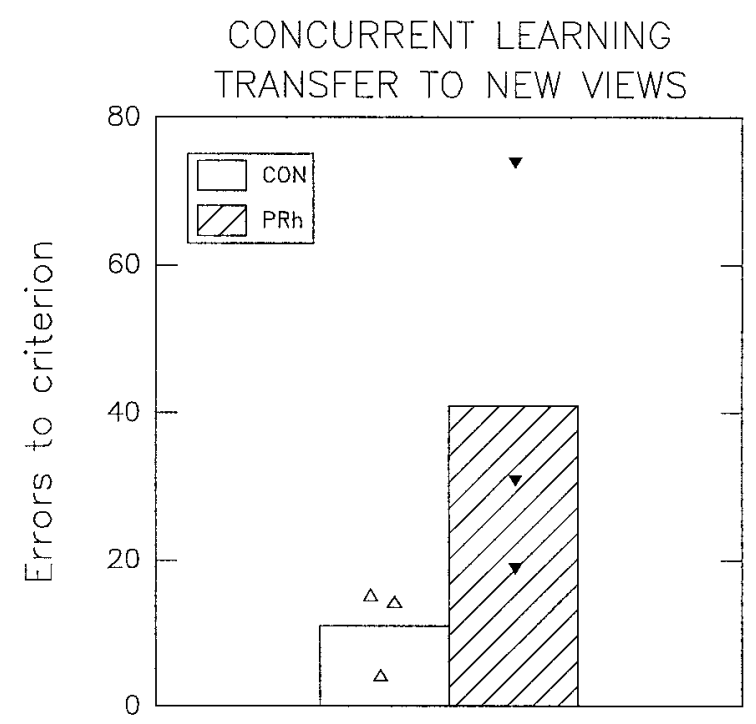

Figure 5. Mean errors to criterion made by each group in stage B of experiment 1 in which the 40 concurrent discrimination learning problems that were learned to criterion in stage A were retested using new views of each of the objects in stage B. Individual scores for each subject are also plotted (triangles). $C O N, n=3 ; P R h, n=3$.

illustrated in Figure 5. The CON group made a mean of 11 errors before attaining criterion on stage $\mathrm{B}$, whereas the PRh group made a mean of 41 errors, which was significantly greater than the number of errors made by the CON group (Wilcoxon rank sum $\mathrm{W}$ test: $\mathrm{W}=15 ; p=0.05$; one-tailed test). Thus the PRh group was significantly impaired relative to the CON group at performing a set of well learned concurrent discrimination problems when the objects were presented in new views.

\section{Experiment 2: identification of familiar objects presented in scenes}

In experiment 2 the subjects were required to learn a set 10 concurrent discriminations problems to criterion between pairs of objects presented in scenes. The subjects were familiar with the objects and with the object-reward contingencies, but they had not experienced these objects presented in the context of digitized images of complex scenes before. Errors to criterion from experiment 2 in which the subjects had to identify familiar objects presented within scenes are illustrated in Figure 6. The CON group made a mean total of 123 errors before attaining criterion on this task, whereas the PRh group made a mean of 246 errors, which was significantly greater than the number of errors made by the CON group (Wilcoxon rank sum $\mathrm{W}$ test: $\mathrm{W}=15 ; p=0.05$; one-tailed test). Thus the PRh group was significantly impaired relative to the $\mathrm{CON}$ group at performing a set of well learned concurrent discrimination problems when each of the pairs of objects was presented in the unfamiliar context of a unique complex scene.

\section{DISCUSSION}

The perirhinal cortex ablations in all three cases were essentially as intended, with only slight and largely unilateral damage to laterally adjacent TE. This inadvertant cortical damage is therefore unlikely to be the cause of the large behavioral effects we report. The behavioral effects of the ablation are also unlikely to be attributable to inadvertent white matter damage. The histological slides show degeneration in the white matter underlying

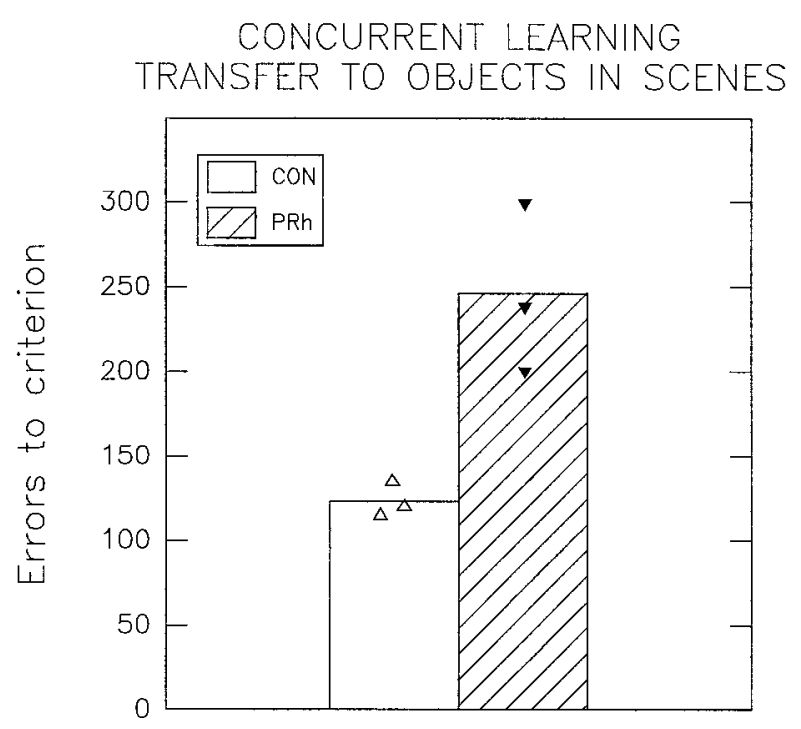

Figure 6. Mean total errors to criterion made by each group in experiment 2 in which 10 well learned concurrent discrimination problems were retested with each of the 10 pairs of objects presented in a context not experienced before, that is, with each pair of objects embedded within a digitized image of an unique scene. Individual scores of each subject are also plotted (triangles). CON, $n=3 ; P R h, n=3$.

the perirhinal cortex, an expected consequence of the cortical ablation; however, little or no damage to the white matter occurred as a result of direct mechanical damage to the white matter during surgery. In previous studies in which perirhinal cortex ablation was combined with ablation of the parahippocampal gyrus or hippocampus (Zola-Morgan et al., 1989, 1993), branches of the posterior cerebral artery that cross the parahippocampal gyrus to supply the inferior temporal gyrus would necessarily have been severed in the course of making the combined ablations, and it remains unclear to what extent the behavioral effects reported in these studies may be attributable to interruption of the blood supply to TE. Indeed, Gaffan and Lim (1991) demonstrated that pial section along the medial boundary of TE, thereby interupting the blood flow in the posterior cerebral artery en route to TE, can affect visual discrimination learning even without producing large infarcts in TE. In contrast, in the course of making perirhinal cortex ablation alone, as in the present study, the blood supply to TE is not interrupted because the perirhinal cortex receives its blood supply principally from the middle cerebral artery. Thus we attribute the behavioral impairments reported in this study to the loss of function of the perirhinal cortex.

Current behavioral evidence is consistent with the hypothesis that there is a deficit in object identification after perirhinal cortex damage. The present experiments test this hypothesis more directly. In experiment 1 subjects learned a concurrent discrimination learning task between 40 pairs of familiar digitized images of objects to criterion. The subjects were then tested on the same problems using similar but previously unseen views of the objects. The subjects with perirhinal cortex ablation were found to be impaired relative to normal controls when tested with the new views (Fig. 5). This provides evidence that perirhinal cortex ablation produces an impairment in object identification and not just in memory. In experiment 2 subjects were required to relearn 10 of the above problems but with each of the 10 pairs of objects presented in the unfamiliar context of a digitized image of a unique complex scene. The subjects with perirhinal cortex 
ablation were found to be impaired relative to normal controls in learning this task to criterion (Fig. 6). This experiment similarly provides evidence that perirhinal cortex ablation impairs object identification and not just memory. We conclude that perirhinal cortex ablation impairs the ability of subjects to identify familiar objects presented in unfamiliar views and the ability of subjects to identify familiar objects presented in the unfamiliar context of complex scenes. These results give considerable support to the hypothesis that the perirhinal cortex has a role in object identification.

We further suggest that the nature of the impairment after perirhinal cortex ablation is a deficit in the ability to process coherent concepts of multiple individual objects, because monkeys with perirhinal or combined perirhinal and entorhinal cortex damage have been shown to be specifically impaired on tasks that require a relatively high level of ability to process coherent concepts of multiple individual objects. The pattern of impairments found in both recognition memory and concurrent discrimination learning after these lesions supports this hypothesis. DMS and DNMS with large stimulus set sizes are impaired, whereas DMS with severely restricted set sizes is unimpaired (Murray et al., 1989; Gaffan and Murray, 1992; Meunier et al., 1993; Eacott et al., 1994; Gaffan, 1994b). Likewise, new postoperative concurrent discrimination learning with small set sizes is unimpaired (Gaffan and Murray, 1992; Eacott et al., 1994; Buckley and Gaffan, 1997), whereas concurrent discrimination learning with larger set sizes is impaired (Buckley and Gaffan, 1997). Other tasks that require relatively high levels of ability to process coherent concepts of multiple individual objects have been found to be selectively impaired after perirhinal cortex ablation. These include concurrent discrimination learning with multiple foils (Buckley and Gaffan, 1997), concurrent discrimination learning with objects presented in different views in each trial (Buckley and Gaffan, 1998a), identification of new views of familiar objects (see experiment 1 ), identification of familiar objects subsequently presented in the context of unfamiliar scenes (see experiment 2), visual stimulus-stimulus association learning (Murray et al., 1993; Buckley and Gaffan, 1998b), and configural learning (Buckley and Gaffan, 1998b). In marked contrast, tasks that do not require a relatively high level of ability to process coherent concepts of multiple individual objects, such as color discrimination (Buckley et al., 1997) and simple spatial discrimination learning (Gaffan, 1994b), are unimpaired. Reassessment of the role of the perirhinal cortex in light of the recent behavioral data therefore provides strong support for the hypothesis that perirhinal cortex damage impairs object identification and is consistent with our suggestion that the deficit lies in the subject's ability to process coherent concepts of multiple individual objects.

This hypothesis is also supported by electrophysiological and anatomical evidence. Saleem and Tanaka (1996) indicated that the perirhinal cortex may receive convergent inputs from multiple widely distributed sites in the ventral part of anterior TE. This suggests that different moderately complex features of objects represented by distant columns in TE (Fujita et al., 1992; Kobatake and Tanaka, 1994; Tanaka, 1996) could be associated together in the perirhinal cortex to represent whole objects. In addition to the prominent inputs from the laterally adjacent unimodal visual areas TE and TEO, the perirhinal cortex also receives projections from diverse unimodal and polymodal areas of association cortex (Suzuki and Amaral, 1994a). The perirhinal cortex is therefore in a position to associate information about stimuli from different modalities, which is consistent with the proposal that the perirhinal cortex has a role in processing coherent concepts of individual objects. Also consistent with this scheme, cells in the perirhinal cortex and some parts of TE (subdivisions TE1 and TE2) (Seltzer and Pandya, 1978), but not in other areas in the inferior temporal cortex, have a higher concentration of cells that show recognition-related responses for specific stimuli in that they respond more strongly to the first than to subsequent presentations of unfamiliar stimuli (Fahy et al., 1993). Furthermore, the perirhinal cortex has recently been functionally doubly dissociated from the middle temporal gyrus (MTG), part of TE (Buckley et al., 1997). Monkeys with MTG ablations were impaired at color discrimination but unimpaired at object recognition memory, whereas monkeys with perirhinal cortex ablation showed the reverse pattern of impairments. This further supports the idea that whereas TE and TEO process information about features of objects, the perirhinal cortex processes knowledge about specific individual objects.

A major defining feature of the perirhinal cortex is its robust interconnections with the hippocampal formation via the entorhinal cortex (Van Hoesen and Pandya, 1975; Insausti et al., 1987; Suzuki and Amaral, 1994b; Suzuki, 1996a,b). In both monkeys and humans the hippocampal system has been implicated in scene memory. Monkeys with fornix transection were severely impaired in a nonspatial scene memory task (Gaffan, 1994c). Such impairment in memory for a discrete event involving a particular object in a particular background may be analogous to the memory impairment for discrete personally experienced events that follows fornix damage in humans (for review, see Gaffan and Gaffan, 1991). Scene memory requires information about object identity to be combined with information about visuospatial relationships between objects; consistent with this, a recent disconnection study showed that interaction between the perirhinal cortex and the fornix is important for scene memory (Gaffan and Parker, 1996). The role of the perirhinal cortex has been differentiated from that of the fornix (Gaffan, 1994b), and in light of such dissociations it has been suggested that there is a loosely hierarchical arrangement of function in the temporal lobe in which the specialization of memory systems is conferred by specialization of their anatomical connections to other structures (Gaffan, 1996; Gaffan and Hornak, 1997a; Buckley and Gaffan, 1998b); for instance, information about isolated features can be associated together and combined with nonvisual object qualities in the perirhinal cortex, and information about individual objects can be combined in the hippocampus with spatial information to represent a spatially organized scene. The present study provides further evidence that the role of the perirhinal cortex can no longer be considered to be restricted to object memory but that it is also involved in object identification. This evidence further erodes the distinction as to whether certain cortical areas should be ascribed as largely perceptual or mnemonic in function. Indeed, it has been suggested that the cortical plasticity that underlies perceptual learning and memory is not fundamentally different (Gaffan, 1996). Evidence that memory retrieval is retinotopically organized (Gaffan and Hornak, 1997b) implies that the function of retinotopic cortical areas, usually thought of as being perceptual in function, may be to maintain a representation of the visual world based on both retinal input and memory. Conversely, it remains to be seen to what extent perceptual impairments toward scenes, in addition to impairments in memory, may follow damage to the hippocampal system.

To conclude, the present experiments have shown that perirhinal cortex ablation impairs the ability of monkeys to identify 
familiar objects presented in new views and impairs the ability of monkeys to identify familiar objects presented in the unfamiliar context of complex scenes. These results demonstrate that the perirhinal cortex has a broader role in visual object memory than was previously known, and along with recent behavioral, antatomical, and electrophysiological data they support the hypothesis that the perirhinal cortex has a role in the visual identification of objects. We suggest that the perirhinal cortex is crucially involved in processing coherent concepts of individual objects. An impairment in this process could underlie the pattern of deficits that follow perirhinal cortex ablation in both visual object recognition memory and visual associative memory.

\section{REFERENCES}

Buckley MJ, Gaffan D (1997) Impairment of visual object discrimination learning after perirhinal cortex ablation. Behav Neurosci 111:467-475.

Buckley MJ, Gaffan D (1998a) Learning and transfer of object-reward associations and the role of the perirhinal cortex. Behav Neurosci 112:1-9.

Buckley MJ, Gaffan D (1998b) Perirhinal cortex ablation impairs configural learning and paired-associate learning equally. Neuropsychologia, in press.

Buckley MJ, Gaffan D, Murray EA (1997) A functional double dissociation between two inferior temporal cortical areas: perirhinal cortex vs. middle temporal gyrus. J Neurophysiol 77:587-598.

Eacott MJ, Gaffan D, Murray EA (1994) Preserved recognition memory for small sets, and impaired stimulus identification for large sets following rhinal cortex ablation in monkeys. Eur J Neurosci 6:1466-1478.

Fahy FL, Riches IP, Brown MW (1993) Neuronal activity related to visual recognition memory: long-term memory and the encoding of recency and familiarity information in the primate anterior and medial inferior temporal and rhinal cortex. Exp Brain Res 96:457-472.

Fujita I, Tanaka K, Ito M, Cheng K (1992) Columns for visual features of objects in monkey inferotemporal cortex. Nature 360:343-346.

Gaffan D (1992) Amnesia for complex naturalistic scenes and for objects following fornix transection in the monkey. Eur J Neurosci 4:381-388.

Gaffan D (1994a) Role of the amygdala in picture discrimination learning with $24 \mathrm{hr}$ intertrial intervals. Exp Brain Res 99:423-430.

Gaffan D (1994b) Dissociated effects of perirhinal cortex ablation, fornix transection and amygdalectomy: evidence for multiple memory systems in the primate temporal lobe. Exp Brain Res 99:411-422.

Gaffan D (1994c) Scene-specific memory for objects: a model of episodic memory impairment in monkeys with fornix transection. J Cognit Neurosci 6:305-320.

Gaffan D (1996) Associative and perceptual learning and the concept of memory systems. Cognit Brain Res 5:69-80.

Gaffan D, Gaffan EA (1991) Amnesia in man following transection of the fornix: a review. Brain 114:2611-2618.

Gaffan D, Lim C (1991) Hippocampus and the blood supply to TE: parahippocampal pial section impairs visual discrimination learning in monkeys. Exp Brain Res 87:227-231.

Gaffan D, Murray EA (1992) Monkeys (Macaca fascicularis) with rhinal cortex ablations succeed in object discrimination learning despite 24-hr intertrial intervals and fail at matching to sample despite double sample presentations. Behav Neurosci 106:30-38.

Gaffan D, Parker A (1996) Interaction of perirhinal cortex with the fornix-fimbria: memory for objects and "object-in-place" memory. J Neurosci 16:5864-5869.

Gaffan D, Hornak J (1997a) Amnesia and neglect: beyond the DelayBrion system and the Hebb synapse. Philos Trans R Soc Lond B Biol Sci 352:1481-1488.

Gaffan D, Hornak J (1997b) Visual neglect in the monkey: representation and disconnection. Brain 120:1647-1652.

Insausti R, Amaral DG, Cowan WM (1987) The entorhinal cortex of the monkey. II. Cortical afferents. J Comp Neurol 264:356-395.

Kobatake E, Tanaka K (1994) Neuronal selectivities to complex object features in the ventral visual pathway of the macaque cerebral cortex. J Neurophysiol 71:856-867.

Malamut BL, Saunders RC, Mishkin M (1984) Monkeys with combined amygdalo-hippocampal lesions succeed in object discrimination learning despite $24 \mathrm{hr}$ intertrial intervals. Behav Neurosci 98:759-769.

Meunier M, Bachevalier J, Mishkin M, Murray EA (1993) Effects on visual recognition of combined and separate ablations of the entorhinal and perirhinal cortex in rhesus monkeys. J Neurosci 13:5418-5432.

Mishkin M, Petri HL (1984) Some implications for the analysis of learning and retention. In: Neuropyschology of memory, Ed 1 (Squire LR, Butters N, eds), pp 287-296. New York: Guilford.

Murray EA, Bachevalier J, Mishkin M (1989) Effects of rhinal cortical lesions on visual recognition memory in rhesus monkeys. Soc Neurosci Abstr 15:342.

Murray EA, Gaffan D, Mishkin M (1993) Neural substrates of visual stimulus-stimulus association in rhesus monkeys. J Neurosci 13:4549-4561.

Overman WH, Ormsby G, Mishkin M (1990) Picture recognition vs. picture discrimination learning in monkeys with medial temporal removals. Exp Brain Res 79:18-24.

Saleem KS, Tanaka K (1996) Divergent projections from the anterior inferotemporal area TE to the perirhinal and entorhinal cortices in the macaque monkey. J Neurosci 16:4757-4775.

Seltzer B, Pandya DN (1978) Afferent cortical connections and architectonics of the superior temporal sulcus and surrounding cortex in the rhesus monkey. Brain Res 149:1-24.

Suzuki WA, Amaral DG (1994a) Perirhinal and parahippocampal cortices of the macaque monkey: cortical afferents. J Comp Neurol 350:497-533.

Suzuki WA, Amaral DG (1994b) Topographic organization of the reciprocal connections between monkey entorhinal cortex and the perirhinal and parahippocampal cortices. J Neurosci 14:1856-1877.

Suzuki WA (1996a) The anatomy, physiology and functions of the perirhinal cortex. Curr Opin Neurobiol 6:179-186.

Suzuki WA (1996b) Neuroanatomy of the monkey entorhinal, perirhinal and parahippocampal cortices: organization of cortical inputs and interconnections with the amygdala and striatum. Semin Neurosci 8:3-12.

Tanaka K (1996) Inferotemporal cortex and object vision. Annu Rev Neurosci 19:109-139.

Van Hoesen GW, Pandya DN (1975) Some connections of the entorhinal (area 28) and perirhinal (area 35) cortices of the rhesus monkey. I. Temporal lobe afferents. Brain Res 95:1-24.

Zola-Morgan S, Squire LR, Amaral DG, Suzuki WA (1989) Lesions of perirhinal and parahippocampal cortex that spare the amygdala and hippocampal formation produce severe memory impairment. J Neurosci 9:4355-4370.

Zola-Morgan S, Squire LR, Clower RP, Rempel NL (1993) Damage to the perirhinal cortex exacerbates memory impairment following lesions to the hippocampal formation. J Neurosci 13:251-265. 